LEONARDO MANNA

Facoltà di Teologia di Lugano, Università Vita-Salute San Raffaele mannal@teologialugano.ch

\title{
IL PENSIERO COME RELAZIONE 0 INTERO SEMANTICO? INTORNO ALLA FILOSOFIA DI LUCIANO FLORIDI
}

In Pensare l'infosfera La filosofia come design concettuale, Luciano Floridi states the value of a correct analysis and philosophical responses within the information age we are living in. The aim of this paper is to suggest several ideas for reflecting on some of Floridi's topics such as the analysis of presence, relationship and semantization. In the second paragraph, after a brief introduction about the author, I will analyse philosophical insights on the relationship between being and determination. In the third paragraph, I am going to suggest the importance of a correct analysis of presence. In the fourth paragraph, a possible analogy between the philosophy of information and a certain form of neo-idealism will be shown. At last, I try to show how the philosophy of information can play a key role in the way we think about reality, but also its possible conceptual risks.

information, semantization, thought, presence

Phenomenology and Mind, n. 20 - 2021, pp. 164-174 DOI: https://doi.org/10.17454/10.17454/pam-2015

https://www.rosenbergesellier.it/eng/journals/phenomenology-and-mind
(C) The Author(s) 2021 
1. La filosofia come primarietà dell'informazione
Luciano Floridi da anni delinea con crescente precisione, competenza e acume speculativo il ruolo fondamentale della filosofia dell'informazione all'interno della contemporaneità, con tutti gli elementi che da questa dipendono: etica, società, privacy, politica, governance, per citare solo alcuni ambiti di applicazione. L'informazione nel nostro tempo ha un ruolo di primaria importanza e, di conseguenza, è imprescindibile la comprensione di questa nel suo statuto ontologico e quindi filosofico. Negli ultimi anni, Floridi sta ampiamente portando avanti il discorso sul valore rivoluzionario dell'informazione ${ }^{1}$ e su come l'uomo contemporaneo in quest'era si stia riconfigurando in rapporto ad essa come inforg. In merito a queste pregevoli e imprescindibili questioni, di cui la filosofia ormai non può assolutamente fare a meno, il testo di Floridi ${ }^{2}$ andrebbe considerato in modo ambivalente come indice riassuntivo rispetto alle pubblicazioni degli ultimi anni dello stesso Floridi e, dall'altro lato, come passo aggiuntivo rispetto a queste. Questo saggio rappresenta un invito ulteriore a prender sul serio la filosofia dell'informazione, vista come conoscenza mediante la quale "negoziamo la corretta tipologia di comunicazione che intratteniamo con il mondo" (p. 100) per comprendere la realtà di esso. Floridi suggerisce che si può divenir "amici della verità, senza essere nemici di Platone" (p. 101), in forza del principio secondo il quale non bisogna identificare colui che conosce con colui che utilizza l'informazione. Da questo presupposto è possibile intravedere tutta l'impostazione costruzionista di Floridi, da cui si apprende che il mondo si configura a partire da chi costruisce l'informazione e non attraverso artefatti eidetici già predisposti. Il filosofo nell'era dell'informazione è un costruttore, un agente poietico oltre che un utente, è sia un produttore che un consumatore. Floridi dimostra che le domande filosofiche sono sempre aperte anche se non ultime e così la filosofia si rivela essere una evoluzione continua di artefatti semantici, di domande aperte. La filosofia è un continuo disegno di concetti, che chiarisce domande, che son sempre aperte, disegnando risposte valide e convincenti. La filosofia, nel suo esser dinamica, è sistematicamente in evoluzione:

1 Riporto solo alcuni dei lavori apparsi recentemente in Italia come: Floridi, L. (2012). La rivoluzione dell'informazione. Torino: Codice; Floridi, L. (2017). La quarta rivoluzione: Come l'infosfera sta trasformando il mondo. Milano: Cortina; Floridi, L. (2009). Infosfera: Etica e filosofia nell'età dell'informazione. Torino: G. Giappichelli; e il recente Floridi, L. (2020). Il verde e il blu: Idee ingenue per migliorare la politica. Milano: Raffaello Cortina Editore.

2 Floridi, L. (2020). Pensare l'infosfera: La filosofia come design concettuale. 
È uno spazio aperto: chiunque può avervi accesso, non importa quale sia il punto di partenza, e il disaccordo vi resta sempre possibile. È anche uno spazio dinamico, poiché quando l'ambiente culturale cambia, la filosofia fa lo stesso ed evolve (Floridi, 2020, p. 54).

L'approccio filosofico è indispensabile per l'accrescimento del capitale semantico legato alla produzione, relazione e comprensione delle informazioni. Il minimalismo, il metodo dei livelli di astrazione e il costruzionismo, nel modo in cui li concepisce Floridi in Pensare l'Infosfera, costituiscono gli strumenti centrali per una corretta filosofia dell'informazione, in cui l'ultimo stadio della riflessione filosofica, su cui va mantenuta aperta la riflessione, deve essere una continua semantizzazione dell'essere.

Floridi si dichiara "costruzionista", e così deve essere la stessa filosofia: costantemente in fase di progettazione in atto di design concettuale, in un contesto in cui il concetto stesso di poiesis viene lentamente identificandosi a quello di noesi:

Se l'autentica conoscenza è conoscenza della natura intrinseca dell'oggetto conosciuto (conoscenza dell'ontologia del conosciuto) e se non sussiste alcuna innata acquisizione di tale prototipo, allora conoscere un fenomeno, un artefatto o, nel nostro caso, conseguire un'informazione ed essere in grado di renderne conto, significa essere capaci di produrre e riprodurre, comporre e scomporre, costruire, montare e smontare tale fenomeno, artefatto o informazione, ovvero di migliorarli o rispondere a domande al loro riguardo, e in ciascun caso per le ragioni corrette. Perché la conoscenza sia possibile, la mimesis deve essere sostituita dalla poiesis (Floridi, 2020, p. 109).

Lo spazio all'interno del quale ogni possibile informazione rimodella la natura dell'uomo come agente informazionale e poietico va compreso superando la dicotomia platonica fra techné ed epistème, ovvero, come ribadisce l'autore, tra sapere pratico e sapere teoretico. Non si tratta quindi solo di comprendere il potenziale fine a sé stesso dell'informazione come ancella della tecnologia, ma al contrario di rendersi conto del valore dell'informazione in rapporto a molteplici campi scientifici nei quali il valore aggiunto sta nella creazione di nuovi ambienti e significati. L'informazione e la comprensione di essa nelle sue dinamiche ha qui il suo ruolo primario.

Per la formulazione di una corretta comprensione della filosofia dell'informazione, il testo di Floridi offre questo tipo di struttura: 1) la rilevanza di una domanda filosofica; 2) l'importanza della conseguente risposta filosofica; 3) la filosofia come disegno concettuale; 4) cinque lezioni tratte dalle conclusioni raggiunte nelle parti precedenti: fissare il corretto livello di astrazione, quali sono le giuste domande filosofiche da porre, riformulare una nuova antropologia filosofica, utilizzare la filosofia dell'informazione per comprendere il mondo contemporaneo e come formare il capitale semantico futuro. Nel nostro intervento ci soffermeremo sui punti 2) e 3), corrispondenti al secondo e terzo capitolo dell'opera in questione, ossia sulle tesi poste da Floridi nel libro in oggetto riguardanti il concetto di presenza e il metodo dei livelli di astrazione $e^{3}$.

3 Per un approfondimento del metodo dei livelli di astrazione proposto da Floridi in modo decisamente più analitico si guardi al suo Floridi, L. (2008). The method of levels of abstraction. Minds and machines, 18(3), 303-329. Lo stesso articolo è stato poi rimaneggiato dallo stesso Floridi ed arricchito con notevoli altri approfondimenti ed inserito nel terzo capitolo della sua opera: Floridi, L. (2013). The philosophy of information. Oxford: Oxford University Press. Ed ancora il metodo dei livelli di astrazione viene sistematizzato nella sua versione originale in inglese, di cui la versione italiana è contenuta nell'opera in questione del presente saggio, ovvero: Floridi, L. (2013). The ethics of information. Oxford: Oxford University Press. 
2. Semantizzazione e determinazione
Floridi è perentorio, sin dall'inizio dell'opera, nel dichiarare l'importanza di mantenere sempre aperta la "semantizzazione dell'essere" (Floridi, 2020, p. 53), non dando troppo peso al punto di partenza, ma stabilendo la rilevanza del disaccordo informato, poiché tale disaccordo è sempre una possibilità. Viene spontaneo, quindi, considerare la semantizzazione, come Floridi converrebbe, in quanto produzione e disegno costante di ontologie, una modellazione sistematica di enti possibili. La produzione, ontologicamente parlando, è sempre un qualcosa di cui possiamo parlare, è sempre legata al soggetto presente, un ente che opera direttamente sulla realtà. Nel saggio di Floridi in questione, non è stata data una definizione di ente, benché questo venga spesso associato ad altri termini come: agente, utente o ente informazionale. Potremmo definire l'ente come l'origo concettuale dal quale ogni domanda e relativa risposta viene formulata e originata. Per una corretta definizione di ente si potrebbe dire simpliciter: quell'elemento minimo di significato da cui ha origine qualsivoglia postulazione. Il primo capitolo si occupa dell'origine di una postulazione e di come questa parta da una corretta formulazione di domande, le quali, assieme alle relative risposte, non sono mai sospese, ma "incorporate in una rete di ulteriori domande" e risposte. Le domande sorgono sempre all'interno di un contesto e, nello specifico, sono sempre lette a un livello di astrazione (LdA) più o meno complesso.

Ebbene, l'ente, in quanto portatore di significato e punto di partenza dal quale è possibile costruire ogni discorso, secondo Floridi non è scisso dal domandare filosofico, non può mai oltrepassare il suo orizzonte. Si noti una analogia con il principio heideggeriano, che lo stesso Floridi descrive come "semantica della presenza o del Dasein" (p. 65). Essere situato sempre all'interno di un determinato ambiente, rimarca a grandi linee l'esser-gettato di cui parla lo stesso Heidegger in Sein und Zeit. Questo trovarsi non è pre-strutturale, non è trascendentale, ma è Geworfenheit, il qualificarsi da parte dell'ente nel suo essere situato, collocato. In questo modo l'esserci di Heidegger, il Dasein, rivoluziona l'approccio alla comprensione fenomenologica di un concetto originario e alla cognizione del mondo inteso nel suo essere semantizzato, sempre a una certa comprensione. Dice Heidegger che "l'esserci è innanzitutto e per lo più presso il "mondo" di cui si prende cura" (Heidegger, 1927, p. 279).

Nella filosofia dell'informazione viene proposta una semantizzazione dell'ente e, così, viene dichiaratamente abbandonata una indagine delle cose ultime o delle domande ultime. Ciò accade perché, per Floridi, la natura di ogni domanda/problema va investigata dal punto di vista della sua complessità e le azioni necessarie per risolverlo/comprenderlo sono sempre determinate dalla complessità dell'analisi che guarda al problema stesso e alle sue risorse: l'orizzonte del problema.

Le domande ultime, quindi domande assolute, per l'autore, sono domande che possono generare solo una quantità enorme di ulteriori domande e risposte. Kantianamente, Floridi porta avanti il principio secondo il quale il mondo noumenico delle cose in sé è inconoscibile, poiché è impossibile da comprendere nella sua interezza, ma in modo molto diverso. La stessa domanda qui non ha senso di porsi. Interrogarsi sull'Essere come concetto di Intero o come termine primo/ultimo è un procedimento che non deve essere metafisicamente compreso o meglio de-finito (ti estì socratico), ma va semantizzato, parafrasando Gustavo Bontadini ${ }^{4}$. Su questa linea possiamo porre ora una netta distinzione fra semantizzare e definire. La definizione dell'Essere è a posteriori e compete all'ente in quanto orizzonte già semantizzato, già dotato di identità e significato. Se l'ente è il punto nodale dal quale partiamo per costituire una

4 Un breve testo, fra i vari, che posso citare di Gustavo Bontadini, che offre importanti spunti per dialogare sui tem trattati è sicuramente: Per una teoria del fondamento, in Bontadini, G. (2009). Metafisica e deellenizzazione. Milano: Vita e pensiero. 
domanda - poi si dirà a un determinato LdA - allora ogni risposta/domanda è costituita da un modello di essa, da una formalizzazione mediante la quale possiamo dire qualcosa.

Secondo questa prospettiva non è possibile dire, filosoficamente, cosa sia una cosa in sé, ma solo se questa cosa che è (ente) funziona in base a uno scopo determinato. Il modello funziona se questo produce uno scopo funzionale all'accrescimento o decremento di significato. Una prima conseguenza che viene spontanea da queste brevi note è che i modelli, di cui possiamo fare esperienza e di cui possiamo parlare, sono finiti. Nello specifico, il modello è in funzione degli osservabili che sono disponibili, lo stesso Floridi definisce il LdA come "un insieme finito, ma non vuoto, di osservabili" (Floridi, 2020, p. 48). Dal modello possiamo ricavare una definizione primitiva di mondo. Più precisamente: ciò che sappiamo, lo sappiamo mediante l'ente (ciò che è), che dà notizia del modello e permette che possa esserci una somma determinata di modelli finiti a cui fa riferimento. Floridi mostra che questa definizione è pluralista e non relativista, in forza del fatto che i modelli possono render conto solo della realtà conoscibile, relativa all'ente e poi a un dato LdA e mai di quella ultima, escatologica o teleologica.

Andando più a fondo, però, questo è possibile solo per mezzo dell'informazione, che consiste noeticamente in dati ben formati e dotati di significato. L'informazione va definendosi come qualcosa che è, di cui possiamo parlare e che è già dotato di significato e sempre inteso in modo determinato, poiché analizzato a un dato LdA.

Possiamo parlare sempre di qualcosa che è in quanto ente, preso nel suo momento speculativo a un determinato LdA, presente e in perenne discussione rispetto al suo esser presente. Uno stato di cose, in quanto è sempre posto in base alle sue risorse e quindi orientato semanticamente, è anche sempre già determinato. Conosciamo i suoi termini, secondo Floridi, in quanto sappiamo di essi mediante quel LdA determinato, che ricordiamo essere dinamico: la complessità è analizzata a seconda del "diversificarsi" della sua posizione.

Citando Deleuze, potremmo dire che "la differenza è lo stato in cui si può parlare della determinazione" (Deleuze, 1968, p. 53) o, meglio, che possiamo definire la presenza dell'ente, come un qualcosa dotato sempre di significato, ma anche sempre soggetto a una modellazione della sua ontologia, della differenziazione concettuale. "D'altro canto, quando determiniamo la differenza come differenza concettuale, crediamo di aver fatto abbastanza per la determinazione del concetto di differenza in quanto tale" (p. 51); questo perché il concetto per Deleuze segue:

la determinazione da un capo all'altro, in tutte le sue metamorfosi, e la rappresenta come pura differenza consegnandola a un fondamento, in rapporto al quale non importa più di sapere se si è davanti a un minimo o a un massimo relativi, davanti a un grande o a un piccolo, davanti a un principio o a una fine, poiché entrambi coincidono nel fondamento come un solo e stesso momento "totale", che è anche quello dello svanire e del prodursi della differenza, quello della sparizione e dell'apparizione (Deleuze, 1968, p. 76).

L'“impegno ontologico" di cui parla Floridi ha questo obiettivo. Formalmente, l'impegno ontologico che l'ente assume porta questo stesso a esser il soggetto del suo diversificarsi: considerando sé e modificando il suo LdA, l'ente, per mantenere il suo statuto di esistente e variabile, dovrà sempre negare la sua posizione ontologica, ma porsi anche a fondamento di essa. Impegnarsi ontologicamente rispetto a uno specifico sistema significa che tale sistema viene modellato, sostituito in favore di un sistema diverso, non per forza qualitativamente migliore o peggiore. La scelta di un determinato LdA permetterà che vi siano quelle informazioni necessarie o osservabili, che utilizzeremo per elaborare il modello. L'osservazione è - secondo 
questa posizione - soggetta alla sua negazione in favore di una osservazione diversa. Ed è possibile affermare ciò a partire dalla affermazione che Floridi fa in merito al sistema, per cui ogni mutazione del sistema corrisponde a una mutazione di stati e viceversa; una continua transizione fra stati. Questi sistemi si determinano dal valore delle proprietà poste in ogni momento di questa loro mutazione.

Risulta indispensabile la tematizzazione relativa a un altro termine adoperato da Floridi: la presenza. L'autore definisce la presenza mostrando i limiti legati a una analisi meramente epistemologica: il fallimento epistemico (FE) è insufficiente per definire una descrizione ben formata delle cose e non permette che si possa costituire la sua negazione o l'assenza di essa. La presenza siffatta può inglobare semanticamente altri spazi come la co-telepresenza e l'assenza - ciò che non è presente - e può assumere solo una connotazione metafisica, aperta a speculazioni su termini e problemi astratti. Se il FE non può definire precisamente l'assenza, Floridi assume come metodo rilevante, approfondendo l'uso degli LdA, l'osservazione di successo (OS).

Floridi parafrasa Quine nella definizione delle modalità di esistenza dei livelli di astrazione e formula lo stesso LdA come il valore di una variabile tipizzata, in cui la necessità di un LdA è a sua volta dipendente da un ulteriore LdA scelto. La presenza, se è dipendente dall'ontologia che scegliamo per analizzare qualsiasi cosa, si pone in ogni analisi possibile come precomprensione oggettiva, ancora, sempre determinata ed immediata. Un ulteriore elemento in accordo con la filosofia heideggeriana, dove l'esserci, il Dasein, è sempre situato, finito e trasmesso.

3. La filosofia come interezza o mediazione della presenza
È in corso, secondo il filosofo dell'informazione, una vera e propria rivoluzione dell'informazione. L'ontologia che nasce a partire da questa rivoluzione, come detto, predispone la filosofia al design come progetto futuro perenne e mediante l'uso dei LdA, Floridi tematizza la collocazione spaziale e temporale della presenza, dimostrando il modo di intendere correttamente la sua localizzazione.

Se la presenza è tutto ciò che è possibile dire del mondo e mediante cui possiamo superare la dicotomia fra presenza epistemologica e presenza dislocata, allora è necessario mostrare in che modo l'analisi della presenza siffatta sia anch'essa soggetta a questa stessa analisi. In primis, Floridi tiene a distinguere l'analisi della presenza (ente) dall'analisi dall'Essere inteso metafisicamente, come qualcosa di scarso interesse per una filosofia forte. Già l'ente, quindi, che si costituisce come fondamento e, come visto sopra, non viene affermato in base alla sua presenza, bensì come mediazione razionale. L'ente informazionale, di cui parla Floridi, viene esibito come "presente", presupponendo che la determinabilità dell'osservabile sia un elemento dialetticamente scisso dalla presenza stessa. L'ente ed il suo contenuto semantico emergono posteriormente alla loro affermazione e perciò dopo la valutazione funzionale della loro esistenza.

Le questioni poste fino ad ora sollevano nuovi quesiti: chi è il subjectum, ovvero il soggetto, che vede questa presenza? A sua volta è una presenza? In che modo si possono postulare concetti al di fuori di questo? Il problema sollevato apre a due vie ulteriori: 1) la presenza è luogo dell'intero semantico oltre il quale non possiamo andare e, pertanto, bisogna ammettere la possibilità che il modello della presenza sia fallibile, in quanto la stessa analisi, poiché è un modello possibile, è soggetta a modificazione; 2) oppure l'analisi della presenza è esterna a essa, tale da supporre che vi sia una presenza, eccedente l'ente analizzato, un ente maggiore che vede l'ente minore, dunque, una analisi dell'oggetto che dice di più dell'oggetto analizzato. Il definiens è maggiore del definiendum.

Questa analisi se posta a un determinato LdA non elimina il problema, ma lo complica. Nel primo caso non è mai possibile uscire dall'analisi parziale del fenomeno, mentre nell'altro caso 
dobbiamo ammettere che l'analisi del fenomeno non è esterna, ma mediata da colui che pone l'analisi. Nel secondo caso, avendo una scissione concettual-oggettuale, l'oggetto analizzato non compete in toto alla presenza, incorrendo in un ingenuo realismo interpretativo. La presenza, essendo sempre in relazione a sé stessa, si pro-getta sempre mediante il trascendimento del suo contenuto. Inoltre, il contenuto è l'oggetto intenzionale e immediato dell'esperienza.

Se la risposta alla prima critica conferma che il problema non abbia senso di porsi, in quanto la risposta è contemplata a un LdA diversificato, si dovrà, in ogni caso, giustificare perché tale diverso LdA può avere valore ontologico super partes, considerato che l'osservato deve esserci oggettivamente a priori dell'analisi, altrimenti non parleremmo di nulla. Ossia, in quanto c'è qualcosa e questo qualcosa è analizzabile se e solo se utilizziamo un determinato LdA con le sue relative proprietà, questo qualcosa è sempre determinato, mediato razionalmente. Possiamo quindi porre questa obiezione: perché questo "qualcosa" non viene definito meglio nella sua essenza, ma continua ad essere proposto a partire dalla sua "presupposizione" e quindi letto già a un determinato LdA?

Se il capitale informazionale semantizza qualsiasi cosa, il discorso di Floridi rischia di diventare un "relativismo costruzionista", in cui ciò che si va a costruire è una catena relazionale di concetti che non riescono mai a de-finirsi, ma solo a dichiararsi estensionalmente. Se si dichiara la non assolutezza del modello (possibilità del modello), allora questo stesso potrà essere aperto, sì, alla sua modificazione ontologica perpetua, alla costante re-ontologizzazione, ma dovrà anche essere disposto ad accettare che la sua stessa valenza può essere soggetta a modificazione, sostituzione o addirittura annullamento, non per forza mediante un artefatto logico similare, bensì attraverso qualcosa di totalmente nuovo, non importa se migliore o peggiore.

Concludendo, se ogni analisi è posta a un determinato LdA e in forza di ciò si afferma la validità di tale analisi, anch'essa a un determinato LdA, allora l'analisi deve ammettere di essere una parziale. Il discorso sulla "osservazione di successo" (OS), posta nel secondo capitolo del saggio in questione, esaspera questa dinamica, poiché ipotizza si possa vedere ciò che è altro dall'analizzante non superando la semplice aporia sopra descritta. Per Floridi il flusso delle informazioni ad un dato LdA permette che un ente sia presente in uno "spazio remoto di osservazione" (che chiama SRO) soltanto se è anche osservato con "successo" ad un determinato LdA in quanto portatore di proprietà (sistema informativo). Tale applicazione degli LdA permette che vi sia una valutazione della presenza e il suo relativo impegno ontologico eludendo così errori di valutazione di portata soggettivistica, cioè: fenomenologici o psicologistici. Se la filosofia dell'informazione, in quanto design, desidera propendere per questa via, si metterà sicuramente sempre in gioco e modificherà il proprio "capitale semantico" Una filosofia radicale dovrebbe riflettere in modo critico il valore della "presenza", così come si è provato molto brevemente a delineare.

"L'unico vero metodo in filosofia è pensare", afferma Floridi (2020, p. 43): questo è il "compito" filosofico, in cui il pensiero viene assimilandosi al concetto di design, pensiero poietico. Ebbene, tornando al concetto di "pensiero", per la tradizione idealista e neoidealista l'esercizio del pensare è stato sempre sinonimo di pensare la Verità oltre che identificato al concetto di metodo e sistema. Il pensiero nella tradizione neoidealista è un pensiero mai scisso dal suo essere attuale, in atto. È possibile trovare svariate consonanze sia teoretiche che metodologiche tra il discorso di Floridi, che segue il concetto di produzione come poiesis e il discorso attualista di Giovanni Gentile secondo il quale c'è analogia tra il l'azione, quel fare che è indispensabile per la conoscenza e il pensiero stesso.

\section{La filosofia come pensiero}


Secondo il filosofo Gentile, mediante il pensiero, che è sempre dinamico e localizzato, è possibile risolvere l'antinomia kantiana; è interessante l'analogia tra l'attualismo gentiliano e l'esercizio filosofico suggerito in Pensare l'infosfera, in cui vi è un obiettivo similare. Floridi afferma, come già detto, che l'errore epistemico sta nella riduzione di ogni possibile analisi del reale alla soggettività. Gentile propone in svariate opere una indagine speculativa su come si possa evitare la fallacia oggettivo-interpretativa e il limite dialettico nell'indagine del reale.

Il filosofo italiano suggerisce che la realizzazione oggettiva di un qualcosa sta nel pensiero di quel qualcosa, vista come operazione consequenziale, ma soggetta alla convinzione che l'alterità rispetto al pensiero stia fuori dalla struttura di questo:

Un pensiero altrui, pur volendolo pensare come altrui, non possiamo pensarlo se non pensandolo come pensiero [...] facendolo nostro. [...] pensare un pensiero (o porre il pensiero oggettivamente) è realizzarlo; ossia negarlo nella sua astratta oggettività per affermarlo in una oggettività concreta (Gentile, 1913, p. 184).

Questo vizio speculativo costituisce una catena di errori basati sulla convinzione che il pensiero possa essere il subjectum per esperire qualcos'altro da sé di qualsivoglia forma. Nel secondo momento speculativo della dialettica gentiliana, e consequenzialmente al primo, la determinazione dell'alterità come comprensione delle proprietà di questa e come ogni possibile altro dal pensiero permette che vi sia una lenta negazione dell'astratto. Il pensiero si riempie della consapevolezza crescente che l'oggetto assume in esso (modellazione):

Questo secondo momento, reso possibile dal primo, se annulla l'attualità del pensiero altrui, o nostro e non più nostro, l'annulla in un nuovo atto di pensiero; per cui l'oggettività nuova [...] conferita a cotesto pensiero [...] è realizzata in funzione del nuovo pensiero, nostro attuale; ed è membro organico dell'unità immanente di questo (Gentile, 1913, p. 185).

Si veda che per Gentile non vi è solo una descrizione logica del processo epistemico, ma vi è anche un processo prasseologico $0^{5}$ è parte della consapevolezza logica nei momenti del pensiero che si realizza la realtà di questo stesso pensiero. Anche per Floridi il raggiungimento/consapevolezza di ogni oggetto è all'interno della prassi speculativa ed è quindi genetico, si evolve con la consapevolezza del capitale semantico che si porta dietro. Oltretutto, non avrebbe senso il tentativo di fuoriuscire da questo orizzonte, poiché se il dicibile è tale all'interno di un orizzonte semantico già definito, allora il pensiero (ente determinato) si configura come designer. Non si tratta, quindi, di voler ridurre tutta la realtà all'Uno del pensiero, ma al contrario mostrare che la totalità del dicibile è tale perché è esperito secondo un modello ontologicamente inscindibile dal pensiero in atto ${ }^{6}$.

5 Per l'importanza di questi concetti legati alla filosofia della prassi è d'obbligo citare sia: Teoria generale dello spirito come atto puro e Genesi e struttura della società, entrambi contenuti in Gentile, G., Severino, E., \& Cicero, V. (2015). L'attualismo. Milano: Bompiani.

6 Oltre alla filosofia gentiliana vi sono stati svariati altri autori nostrani a partire dai quali si son sviluppate riflessioni sulle innumerevoli implicazioni problematiche del concetto di presenza, del rapporto fra pensiero e realtà o del pensiero ed i suoi limiti. Ne cito solo alcuni relativi al panorama italiano che ritengo rilevanti: Calogero, G. (2015). Filosofia del dialogo. Brescia: Morcelliana; Carabellese, P. (1948). Critica del concreto. Firenze: Sansoni; Spirito, U. (1948). Il problematicismo. Firenze: Sansoni; Bacchin, G. R., \& In Castegnaro, G. (2017). Theorein. Roma: Aracne; Severino, E. (1981). La Struttura originaria. Milano: Adelphi. 
Floridi sostiene che "il capitale semantico digitale inizia a fare la differenza nel nostro stesso processo di semantizzazione” (Floridi, 2020, p. 117), il capitale semantico è quindi in uno stato procedurale di affermazione, in una dynamis come atto, come movimento. Anche per Gentile non si può mai trascendere l'atto, non si può guardare come un "fatto" qualcosa di compiuto, visto dal di fuori del pensiero. Un'altra analogia gentiliana assimilabile con l'analisi che Floridi propone del processo di semantizzazione riguarda la dichiarazione delle risorse disponibili per il compimento delle operazioni utili alla risoluzione di problemi. Se questo processo viene visto come energheia illimitata, per la produzione di domanda aperte, al di fuori delle quali non è possibile uscire se non come accordo informato, allora il pensiero come interfaccia andrebbe inteso, come sosteneva anche Gentile, in quanto fare, come atto creativo. L'attualista italiano, contestando l'impostazione metafisica, propose di evitare l'errore trascendente dell'assoluto come un qualcosa di già “dato". Ancora, Floridi, così come Gentile, condivide l'errore della tesi platonica secondo la quale un approccio valido alla conoscenza non può essere mimesis, ma deve essere poiesis, quindi non imitazione di un già dato, ma produzione di artefatti. Inoltre, per Gentile l'essere non può negarsi dialetticamente in favore di un altro essere perfettibile, l'essere e la presenza non possono migliorarsi col fine di corrispondere ad un vero già dato. Gentile contesta della dialettica platonica esattamente questa ammissione, in cui la realtà che si vuol conoscere è presupposta al pensiero e con esso la concezione della natura e dell'eternità delle idee. Per Gentile quella positività delle idee, che Platone ambiziosamente cercava, sta nella realizzazione della realtà di cui l'idea stessa è principio. Chiarificando, se l'idea è comprensione della "cosa" allora deve essere prodotta (poiesis) dall'idea stessa. Direbbe Gentile che "il pensiero che è vero pensiero, deve generare l'essere di cui è pensiero" (Gentile, 1916, p. 98), in questo modo per l'attualista si può scorgere il vero significato del cogito cartesiano. In Gentile emerge con forza l'importanza di una costruzione genetica e poietica della storia.

Una ulteriore analogia può esser trovata tra l'accumulo del cosiddetto "capitale semantico" di cui parla Floridi e nel come Gentile intende l'analisi della realtà mediante ciò che chiama "autocoscienza". Il sapere genetico della storia della filosofia, che Floridi definisce "progetto umano [corsivo suo]", rasenta quel "momento dialettico" come "incremento della nostra intelligenza" (p. 253) di cui Gentile parla in Teoria generale dello spirito come atto puro. L'intelligenza come capitale semantico per l'attualista non è già acquisita, tale per cui non necessita di attività ulteriori, ma abbisogna di costante incremento (consapevolezza) per diventare "nuovo sapere" (p. 253). Passato e futuro divengono reali e concreti se visti da quell'intelligenza che li rende attuali, dal pensiero in atto ${ }^{7}$.

L'esercizio del pensare l'atto del domandare come esercizio rigoroso e come realtà integrale può essere utilizzato in forza del superamento del pensiero come delimitazione ad un esclusivo contesto di riferimento o ad una interpretazione della domanda, ad una interfaccia possibile. Andrebbe considerata la forza speculativa in seno all'ontologia del domandare, come autocoscienza, come indispensabilità del dato, quindi come posizione irriducibile ed imprescindibile.

Quella di Floridi è senza dubbio una filosofia democratica e pluralista, che riesce a creare un ponte fra innumerevoli discipline, riuscendo ad evitare un certo vizio logico verificazionista, un approccio realista o empirista delle diverse filosofie contemporanee, che si interrogano sul corretto modo di orientarsi all'oggetto speculativo.

\section{La filosofia come rischio: domanda e risposta}

7 Riguardo all'Atto gentiliano come metodo e come problema, oltre che un breve approfondimento riguardante il neoidealismo gentiliano e gli sviluppi successivi nella filosofia italiana si potrebbe consultare il recente volume Manna, L., \& Iosi, M. (2018). L'eredità di Giovanni Gentile nella filosofia italiana.Roma: Aracne. 
Il suo scopo sembra esser quello di riportare sotto un'unica bandiera la filosofia nel suo statuto primario, come conoscenza e come primato del pensiero. Ciò che emerge dal discorso di Floridi non è il design come fine ultimo, ma come nuovo fine da cui iniziare a comprendere i limiti che la filosofia odierna ha assimilato e mantenuto dopo vizi postmoderni, $\mathrm{d}$ o altre forme di pensiero riduzionista.

La filosofia dell'informazione offre una valida prospettiva rispetto a un modo di porre domande filosofiche, esente da errori empirico-logici, ma mantiene altresì il rischio di fermarsi solo all'inizio della sua formulazione principale: nella comprensione della domanda e della risposta. Nonostante lo stesso Floridi a più riprese dichiari il suo come progetto iniziale di filosofia dell'informazione (p.141). Poiché l'errore è insito nel cattivo domandare, nella formulazione del principio del rispondere c'è un importante bivio da tenere in considerazione. La filosofia della tradizione, da Aristotele a Kant, passando per Hegel o dalla filosofia italiana neoidealista o neotomista, ha mantenuto comunque alto il valore della Verità come fine primo ed ultimo. Inoltre, il bivio che si presenta nel discorso di Floridi può essere pericoloso, perché può portare la filosofia a riscoprirsi forte a partire da ciò che già possiede e a ricoprire il buono della tradizione del pensiero aletico. L'alternativa è che questo sistema possa divenire pura modellazione di concetti, col rischio di trasformarsi in pura relazionalità e portare l'oggetto stesso dell'indagine a costituirsi come catena concettuale indefinita. Una filosofia forte deve dare risposte assiologiche per mantenere il suo statuto filosofico, non deve temere, al contrario di quanto Floridi sostiene, di parlare delle Verità ultime.

La Verità nella tradizione filosofica dai Greci al nostro tempo dovrebbe ancora essere tematizzata intorno al concetto di intero. Proporre una visione dell'intero semantico democraticamente valido, ma mai de-terminato, conduce il pensiero all'autoreferenzialità del suo pro-getto, senza mai costituirsi fattualmente. Non è sbagliato partire dal valore che ogni risultato è progressione. Anche Vico e Croce condividerebbero la virtù genetica e dinamica del pensiero, vedrebbero la progressione storica, orientata al futuro dell'accrescimento come punto di partenza continuo per una costruzione più forte del pensiero.

Ciò che potrebbe apparire come errato dell'immagine che Floridi dà del pensiero è però questo assunto recuperato dalla logica e dalle teorie computazionali: la presupposizione che ogni risultato sia un risultato aperto al dubbio e alla sua riformulazione/costruzione/orientamento. In questo la sua filosofia incorre nel pericolo di diventare l'ultimo stato di una filosofia del possibile, una filosofia aperta solo alle domande, ma mai alle risposte.

\section{RIFERIMENTI BIBLIOGRAFICI}

Bacchin, G. R., \& In Castegnaro, G. (2017). Theorein. Roma: Aracne; Bontadini, G. (2009). Metafisica e deellenizzazione. Milano: Vita e pensiero; Calogero, G. (2015). Filosofia del dialogo. Brescia: Morcelliana; Carabellese, P. (1948). Critica del concreto. Firenze: Sansoni; Deleuze, G., \& Guglielmi, G. (ed.) (1971). Differenza e ripetizione. Collezione di testi e di studi. Bologna: Il mulino; Floridi, L. (2008). The method of levels of abstraction. Minds and machines, 18(3), 303-329. DOI:10.1007/s11023-008-9113-7 Floridi, L. (2009). Infosfera: Etica e filosofia nell'età dell'informazione. Torino: G. Giappichelli; Floridi, L. (2012). La rivoluzione dell'informazione. Torino: Codice; Floridi, L. (2013). The ethics of information. Oxford: Oxford University Press. Floridi, L. (2013). The philosophy of information. Oxford: Oxford University Press. Floridi, L. (2017). La quarta rivoluzione: Come l'infosfera sta trasformando il mondo. Milano: Cortina; Floridi, L. (2020). Il verde e il blu: Idee ingenue per migliorare la politica. Milano: Raffaello Cortina Editore; 
Floridi, L. (2020). Pensare l'infosfera: La filosofia come design concettuale.

Gentile, G., Severino, E., \& Cicero, V (eds.). (2015). L'attualismo. Milano: Bompiani;

Gentile, G. (1954). La riforma della dialettica Hegeliana. Firenze: Sansoni;

Gentile, G. (1987). Teoria generale dello spirito come atto puro. Firenze: Le Lettere;

Heidegger, M., \& In Chiodi, P (ed.). (1969). Essere e tempo: L'essenza del fondamento. Torino:

Unione Tip. Editrice Torinese;

Manna, L. (2018). L'eredità di Giovanni Gentile nella filosofia italiana. Roma: Aracne;

Severino, E. (1981). La Struttura originaria. Milano: Adelphi;

Spirito, U. (1948). Il problematicismo. Firenze: Sansoni. 